Food consumption and nutrient intake in relation to denture use in 55-to 84-year-old men and women -Results of a population based survey

Jauhiainen, L.

2017-05

Jauhiainen , L, Mannisto , S , Ylostalo , P , Vehkalahti , M , Nordblad , A , Turunen , A W \& Suominen, $A \mathrm{~L} 2017$, ' Food consumption and nutrient intake in relation to denture use in 55-to 84-year-old men and women -Results of a population based survey ', Journal of nutrition, health \& aging , vol. 21 , no. 5 , pp. 492-500 . https://doi.org/10.1007/s12603-016-0793-z

http://hdl.handle.net/10138/236798

https://doi.org/10.1007/s12603-016-0793-z

publishedVersion

Downloaded from Helda, University of Helsinki institutional repository.

This is an electronic reprint of the original article.

This reprint may differ from the original in pagination and typographic detail.

Please cite the original version. 


\title{
FOOD CONSUMPTION AND NUTRIENT INTAKE IN RELATION TO DENTURE USE IN 55- TO 84-YEAR-OLD MEN AND WOMEN -RESULTS OF A POPULATION BASED SURVEY
}

\author{
L. JAUHIAINEN ${ }^{1,2}$, S. MÄNNISTÖ3 ${ }^{3}$ P. YLÖSTALO ${ }^{1,4,5,6}$, M. VEHKALAHTI $^{7}$, A. NORDBLAD ${ }^{8}$, \\ A.W. TURUNEN, A.L. SUOMINEN $N^{1,6,9,10}$
}

\begin{abstract}
1. Institute of Dentistry, University of Eastern Finland, Kuopio, Finland; 2. Department of Oral and Maxillofacial Surgery, North Karelia Central Hospital and Honkalampi Centre, Joensuu, Finland; 3. Department of Health, National Institute for Health and Welfare, Helsinki, Finland; 4. Institute of Dentistry, University of Oulu, Oulu, Finland; 5. The Medical Research Center Oulu, Oulu University Hospital and University of Oulu, Finland; 6. Department of Oral and Maxillofacial Surgery, Kuopio University Hospital, Kuopio, Finland; 7. Department of Oral and Maxillofacial Diseases, Faculty of Medicine, University of Helsinki, Helsinki; 8. Ministry of Social Affairs and Health, Helsinki, Finland; 9. The Living Environment and Health Unit, National Institute for Health and Welfare, Kuopio, Finland; 10. The Health Monitoring Unit, National Institute for Health and Welfare, Helsinki, Finland. Corresponding author: Anna Liisa Suominen, University of Eastern Finland, Institute of Dentistry, Kuopio campus, P.O. Box 1627, FI-70211 Kuopio, Finland, E-mail: liisa.suominen@uef.fi, Fax: 040355 2442,Tel: 0403552442
\end{abstract}

\begin{abstract}
Objective: To study differences in consumption of foods and intake of nutrients attributable to denture status. Design, setting and participants: Data from a cross-sectional, nationally representative Health 2000 Survey, subjects aged 55-84 years $(n=2,241)$. Measurements: Denture status (edentulous with full dentures, own dentition with removable dentures, own dentition with no removable dentures) was used as an explanatory variable. The consumption of foods and intake of nutrients was used as an outcome variable and was measured using a validated Food Frequency Questionnaire. Results: Denture status associated with food choices. Full denture wearers consumed less vegetables $(\mathrm{p}=0.013$ among men and $\mathrm{p}=0.001$ among women) and fruits $(\mathrm{p}=0.001$ among women), more sugary products $(\mathrm{p}=0.012$ among men and $\mathrm{p}=0.008$ among women $)$, and their balance in fatty acids was less favourable than among dentate participants. Among dentate participants, the differences between the two groups were small and statistically significant differences were seen mostly in women. Conclusions: Wearing full dentures appears to be associated with unhealthier food choices, lower consumption of some foodstuffs and lower intake of certain nutrients when compared to the food choices of dentate persons.
\end{abstract}

Key words: Denture status, food consumption, national survey, nutrient intake, older people.

\section{Introduction}

There are only a few population-based studies about interrelations between oral health status, food consumption and nutrient intake, but the findings emerging from these studies are quite similar, i.e. the lack of one's own natural teeth is associated with the ability to eat hard or difficult to eat foods (1, $2,3)$. It has also been observed that there are different nutrient concentrations in serum according whether a person has his/ her own natural teeth or not. For example, plasma retinol and plasma ascorbate concentrations have been reported to be lower among edentate than dentate individuals $(1,4,5)$.

On the other hand, results from earlier studies are contradictory regarding differences between dentate individuals and denture wearers in terms of food consumption and nutrient intake. Some studies do not report any major differences in dietary pattern (6) or nutrient intake (7) according to denture status while others report differences not only in the consumption of hard to chew foods (3), but also in nutrient intakes and general food consumption between individuals with or without dentures $(8,9)$.

At the moment, there are few studies of the relationship between dental status and general health. In these studies edentulousness without dentures was reported to associate with higher risk of decreased physical ability and malnutrition when compared to having one's natural teeth. In these studies the findings about the role of edentulousness without dentures in relation to mortality was contradictory $(10,11)$.

Currently, there is insufficient knowledge about the interrelation between the use of partial denture in dentate people and diet (12) and this interrelation has not been studied in the population-based studies mentioned above $(1,3,4)$. The aim of this study was to examine whether denture status (edentulous with full dentures, own dentition with removable dentures, own dentition with no removable dentures) among men and women would be associated with consumption of foods and intake of nutrients in a middle-aged and elderly Finnish population.

\section{Material and methods}

This study was based on data from the nationally representative Health 2000 Survey implemented in 20002001 by the National Institute for Health and Welfare (THL) (former the National Public Health Institute (KTL) of Finland). The survey was conducted in whole Finland using the twostage stratified cluster sampling design. People aged 80 or over were oversampled with a double sampling fraction. The sampling frame was regionally stratified according to the five university hospital regions, each containing roughly one million 


\section{JNHA: NUTRITION}

inhabitants. After this, 80 health center districts were selected among these university hospital regions. The participants were randomly selected among health center districts. The main sample concerning adults aged 30 years and over comprised of 8028 individuals who lived either at home or at institutions in mainland of Finland (13). The present study was restricted to participants aged $55-84$ years $(n=2,241)$ because among the younger ones, number of those who had lost teeth or wore removable dentures was very low (14). All participants of this study filled in a food frequency questionnaire (FFQ), attended a clinical oral health examination and an interview where background information was collected. In the study group, $43 \%$ of participants were men and $57 \%$ were women.

Each participant gave their written, informed consent. Approval of the Ethics Committee of Epidemiology and Public Health in the Hospital District of Helsinki and Uusimaa was also obtained.

\section{Information of dietary variables}

Food consumption was measured using a validated Food Frequency Questionnaire (FFQ) (15). FFQ included 128 food items, which allowed assessment of the whole diet over the previous 12 months. Nine frequency categories of the use of foods ranged from never or rarely to six or more times per day. The portion sizes were fixed, and if possible, specified using natural units (e.g. cups of coffee). The food consumption was converted to grams per day by multiplying the frequency of food consumption by fixed portion sizes. The ingredients of mixed foods were broken down into their components as were the contents of different nutrients in food items to allow them to be estimated using Fineli ${ }^{\circledR}$, a Finnish food consumption database. The daily energy intake (in kcal/day) and the proportions of fats, carbohydrates and proteins in percentages were calculated and they were used in these analyses as continuous variables.

The FFQ was handed to participants in connection with the health examination or home health examination. Participants filled in the questionnaire at their homes without an interviewer, which does not let us know the actual person filling in the questionnaire. However, the questionnaire was introduced to each participant and the filling instructions were reviewed together with them. A nutritionist looked through all forms at the THL and if there was some written information to declare the persons cognitive capacity, it was taken in account at this point. Unreliably filled questionnaires were not used in the analyses (16).

\section{Clinical oral health examination}

The examination of clinical oral health, performed as part of the general health examination, took about 15 minutes (13). It was carried out in a dental chair with a headlamp as the light source and using a mouth mirror, fibre optics and a WHO periodontal probe. The presence of teeth was recorded according to the presence or absence of a tooth taking into account all teeth and tooth remnants visible and tactile in the mouth. The presence of removable dentures was subdivided into upper or lower ones by jaw. In this study, denture status was categorised into three groups: edentulous with full dentures (=full denture wearers), dentate with removable dentures and dentate with no removable dentures.

\section{Interview data}

The interviews gathered information on socioeconomic circumstances, living arrangements, self-reported oral health, and cognitive function. The level of education was assessed using information on formal schooling and vocational training. In these analyses, education was categorised into three classes: basic, intermediate and higher education. Those with no formal vocational training or upper secondary education were classified as having basic education; those who had completed vocational training or passed the matriculation examination were considered as having intermediate education and those with higher education had degrees or diplomas from higher vocational institutions, polytechnics and universities. Marital status was classified into two classes: married or cohabiting vs. single, divorced or widowed. Living arrangements were categorised into two classes: those living at home vs. those living in a nursing home or an institution. Self- perceived oral health was measured with the question: "Is the condition of your teeth and mouth at present, (A) good; (B) fairly good; (C) moderate; (D) fairly poor; (E) poor". In the analyses the categories (A) and (B) were combined as were categories (C),(D) and (E). The participants were also asked whether they had suffered from toothache during the past 12 months or had experienced other problems related to their teeth or dentures (yes/no); whether they were able to eat dry bread / biscuits without simultaneously drinking water (yes/no), or whether they were able to chew hard or tough food (such as rye bread) (A) without difficulty; (B) with difficulty or (C) not at all. A shortened version of the Mini Mental State Examination (MMSE) (sum score: mean/13.7, SE, $\min / 0, \max / 16$ ) was used to measure cognitive function (17).

\section{Statistics}

Due to the two-stage stratified cluster sample design and in order to correct the effects of non-response and over-sampling people aged 80 years or older, SAS Callable SUDAAN software was used. Means of consumption of food items and intake of nutrients with $95 \%$ confidence intervals $(95 \% \mathrm{CI})$ were calculated according to denture status. Linear regression was used to first examine weighted but unadjusted associations between denture status and food consumption and nutrient intake. In these analyses, the food items or nutrient intakes were used as the outcome variables and denture status as the explanatory variable. Associations were first adjusted for age as continuous and energy intake, next for age as continuous, energy intake, marital status, level of education and living arrangements, and finally for age as continuous, energy intake, 
Table 1

Distribution of the study subjects according to gender and denture status*

\begin{tabular}{|c|c|c|c|c|c|c|c|c|}
\hline \multirow[b]{2}{*}{ (missing value) } & \multicolumn{4}{|c|}{$\operatorname{MEN}(n=911)$} & \multicolumn{4}{|c|}{ WOMEN (n=1,215) } \\
\hline & $\begin{array}{c}\text { Dentate, no } \\
\text { removable } \\
\text { dentures }\end{array}$ & $\begin{array}{c}\text { Dentate with } \\
\text { removable } \\
\text { dentures }\end{array}$ & $\begin{array}{l}\text { Edentulous } \\
\text { / Full den- } \\
\text { tures }\end{array}$ & ALL & $\begin{array}{c}\text { Dentate, no } \\
\text { removable } \\
\text { dentures }\end{array}$ & $\begin{array}{c}\text { Dentate with } \\
\text { removable } \\
\text { dentures }\end{array}$ & $\begin{array}{l}\text { Edentulous } \\
\text { / Full den- } \\
\text { tures }\end{array}$ & ALL \\
\hline $\operatorname{ALL}(\%)$ & 47 & 31 & 22 & & 39 & 31 & 30 & \\
\hline \multirow[t]{2}{*}{ Age (mean) } & 63.2 & 64.9 & 69.0 & 65.0 & 63.3 & 66.1 & 69.9 & 66.1 \\
\hline & & $<0.001^{1}$ & & & & $<0.001^{1}$ & & \\
\hline \multirow[t]{2}{*}{ Number of teeth (mean) } & 23.2 & 10.3 & 0 & 14.1 & 23.7 & 10.3 & 0 & 12.4 \\
\hline & & $<0.001^{1}$ & & & & $<0.001^{1}$ & & \\
\hline \multicolumn{9}{|l|}{ Education (\%) } \\
\hline Basic & 46 & 60 & 80 & 58 & 44 & 67 & 83 & 63 \\
\hline Intermediate & 29 & 27 & 17 & 26 & 25 & 20 & 15 & 20 \\
\hline Higher & 25 & 13 & 3 & 16 & 31 & 13 & 2 & 17 \\
\hline$(\mathrm{n}=7)$ & & $<0.001^{3}$ & & & & $<0.001^{3}$ & & \\
\hline \multicolumn{9}{|l|}{ Marital status (\%) } \\
\hline Married/cohabiting & 80 & 80 & 77 & 79 & 63 & 53 & 48 & 55 \\
\hline Single/separated/divorced & 20 & 20 & 23 & 21 & 37 & 47 & 52 & 45 \\
\hline$(\mathrm{n}=5)$ & & $0.575^{3}$ & & & & $<0.001^{3}$ & & \\
\hline \multicolumn{9}{|l|}{ Living arrangements (\%) } \\
\hline Home & 100 & 99 & 96 & 98 & 98 & 99 & 98 & 98 \\
\hline Service flat/institution & 0 & 1 & 1 & 2 & 2 & 1 & 2 & 2 \\
\hline$(\mathrm{n}=8)$ & & $0.059^{3}$ & & & & $0.602^{3}$ & & \\
\hline \multicolumn{9}{|l|}{ Self-perceived oral health (\%) } \\
\hline Good/fairly good & 62 & 51 & 63 & 59 & 71 & 52 & 63 & 62 \\
\hline Intermediate/poor & 38 & 49 & 37 & 41 & 29 & 48 & 37 & 38 \\
\hline$(\mathrm{n}=18)$ & & $0.042^{3}$ & & & & $<0.001^{3}$ & & \\
\hline \multicolumn{9}{|l|}{ Pain/discomfort during the past 12 months (\%) } \\
\hline Yes & 30 & 38 & 19 & 30 & 37 & 34 & 24 & 31 \\
\hline No & 70 & 62 & 81 & 70 & 67 & 66 & 76 & 69 \\
\hline$(\mathrm{n}=5)$ & & $<0.001^{3}$ & & & & $0.006^{3}$ & & \\
\hline \multicolumn{9}{|l|}{ Able to eat dry bread/biscuits (\%) } \\
\hline Yes & 90 & 88 & 79 & 86 & 89 & 90 & 71 & 85 \\
\hline No & 10 & 12 & 21 & 21 & 11 & 10 & 29 & 15 \\
\hline$(\mathrm{n}=16)$ & & $<0.001^{3}$ & & & & $<0.001^{3}$ & & \\
\hline \multicolumn{9}{|l|}{ Problems in chewing (\%) } \\
\hline None & 88 & 75 & 58 & 77 & 87 & 74 & 64 & 76 \\
\hline Minor & 8 & 23 & 31 & 18 & 11 & 21 & 29 & 19 \\
\hline Major & 4 & 2 & 11 & 5 & 2 & 6 & 7 & 5 \\
\hline$(\mathrm{n}=11)$ & & $<0.001^{3}$ & & & & $<0.001^{3}$ & & \\
\hline $\begin{array}{l}\text { Cognitive function }\left(\mathrm{MMSE}^{4} \text { sum score,- }\right. \\
\text { mean) }\end{array}$ & 14.1 & 13.8 & 13.1 & & 14.1 & 13.7 & 13.3 & \\
\hline$(\mathrm{n}=151)$ & & $<0.0012$ & & & & $<0.0012$ & & \\
\hline
\end{tabular}

* p-values refer to Kruskal-Wallis 1. ANOVA; 2.or chi square; 3. tests between denture status and the variables listed; 4. A shortened version of the Mini Mental State Examination 


\section{JNHA: NUTRITION}

Table 2

Weighted means of food consumption (g/day) with $95 \%$ confidence intervals $(95 \% \mathrm{CI})$ by denture status in men $(\mathrm{n}=911) *$

\begin{tabular}{|c|c|c|c|c|c|c|c|c|c|c|}
\hline \multirow[t]{2}{*}{ FOOD ITEM } & \multicolumn{2}{|c|}{$\begin{array}{l}\text { Dentate, no removable } \\
\text { dentures }(n=422)\end{array}$} & \multicolumn{2}{|c|}{$\begin{array}{l}\text { Dentate with removable } \\
\text { dentures }(\mathrm{n}=\mathbf{2 8 6})\end{array}$} & \multicolumn{2}{|c|}{$\begin{array}{l}\text { Edentulous with full } \\
\text { dentures }(n=203)\end{array}$} & \multirow[b]{2}{*}{ p-value ${ }^{1}$} & \multirow[b]{2}{*}{ p-value ${ }^{2}$} & \multirow[b]{2}{*}{ p-value ${ }^{3}$} & \multirow[b]{2}{*}{ p-value ${ }^{4}$} \\
\hline & g/day & $95 \% \mathrm{CI}$ & g/day & $95 \% \mathrm{CI}$ & g/day & $95 \% \mathrm{CI}$ & & & & \\
\hline Cereals & 200 & $190-210$ & 200 & 190-210 & 200 & $190-220$ & 0.733 & 0.468 & 0.440 & 0.654 \\
\hline Rye & 55 & $51-59$ & 53 & $50-57$ & 51 & $46-55$ & 0.384 & 0.230 & 0.095 & 0.159 \\
\hline Wheat & 79 & $74-83$ & 82 & $76-88$ & 84 & $76-91$ & 0.437 & 0.812 & 0.825 & 0.870 \\
\hline Hard bread & 6.7 & $5.5-7.9$ & 7.3 & $5.5-9.1$ & 8.5 & $6.3-10.6$ & 0.295 & 0.311 & 0.234 & 0.221 \\
\hline Vegetables & 260 & $240-280$ & 260 & $230-280$ & 210 & $180-230$ & 0.003 & $<0.001$ & 0.002 & 0.013 \\
\hline Root vegetables and tubers & 52 & $48-57$ & 54 & $49-60$ & 52 & $44-60$ & 0.816 & 0.659 & 0.655 & 0.013 \\
\hline Potatoes & 190 & $170-200$ & 190 & $180-210$ & 210 & $190-230$ & 0.068 & 0.111 & 0.229 & 0.220 \\
\hline Fruits & 170 & $150-190$ & 150 & $140-170$ & 130 & $110-150$ & 0.012 & 0.016 & 0.114 & 0.080 \\
\hline Citrus & 34 & $28-40$ & 28 & $22-34$ & 23 & $16-30$ & 0.035 & 0.103 & 0.554 & 0.676 \\
\hline Apples & 88 & $76-100$ & 80 & $67-92$ & 55 & $46-65$ & 0.002 & 0.002 & 0.005 & 0.008 \\
\hline Berries & 28 & $26-31$ & 34 & $31-38$ & 32 & $28-36$ & 0.028 & 0.111 & 0.113 & 0.105 \\
\hline Juice & 65 & $56-73$ & 65 & $54-75$ & 48 & $37-60$ & 0.065 & 0.100 & 0.485 & 0.618 \\
\hline Fish, fish products, crustaceans and molluscs & 49 & $45-53$ & 59 & $53-64$ & 50 & $45-55$ & 0.006 & 0.013 & 0.018 & 0.004 \\
\hline Meat products & 170 & $160-170$ & 170 & $160-180$ & 160 & $150-170$ & 0.442 & 0.734 & 0.745 & 0.741 \\
\hline Red meat & 92 & $87-98$ & 98 & $92-100$ & 91 & $83-99$ & 0.352 & 0.680 & 0.712 & 0.853 \\
\hline Beef & 27 & $25-29$ & 29 & $26-31$ & 27 & $23-30$ & 0.641 & 0.896 & 0.662 & 0.591 \\
\hline Pork & 53 & $50-56$ & 57 & $54-61$ & 53 & $48-57$ & 0.159 & 0.241 & 0.219 & 0.442 \\
\hline Sausages & 42 & $37-47$ & 44 & $38-50$ & 46 & $39-53$ & 0.619 & 0.702 & 0.887 & 0.761 \\
\hline Poultry & 22 & $19-25$ & 20 & $17-23$ & 14 & $12-17$ & 0.001 & 0.019 & 0.144 & 0.105 \\
\hline Eggs & 28 & $26-30$ & 31 & $28-34$ & 32 & $28-37$ & 0.227 & 0.467 & 0.523 & 0.421 \\
\hline Milk & 360 & $330-380$ & 390 & $350-420$ & 420 & $390-460$ & 0.024 & 0.314 & 0.658 & 0.981 \\
\hline Soured milk products & 1700 & $150-190$ & 160 & $140-190$ & 180 & $150-210$ & 0.546 & 0.497 & 0.440 & 0.498 \\
\hline Cheese & 34 & $31-38$ & 31 & $28-35$ & 23 & $20-77$ & $<0.001$ & 0.001 & 0.004 & 0.003 \\
\hline Sugary products & 31 & $29-33$ & 35 & $32-38$ & 41 & $37-46$ & $<0.001$ & $<0.001$ & 0.001 & 0.012 \\
\hline Beverages (-alcohol) & 1400 & $1400-1500$ & 1500 & $1400-1600$ & 1400 & $1300-1500$ & 0.117 & 0.368 & 0.435 & 0.454 \\
\hline \multicolumn{11}{|l|}{ Fat and fat products } \\
\hline Butter and butter spread & 11 & $11-12$ & 13 & $12-14$ & 14 & $13-15$ & 0.002 & 0.033 & 0.175 & 0.251 \\
\hline \multicolumn{11}{|l|}{ Margarine and fat spread } \\
\hline$(\mathrm{FAT})$ & 5.4 & $4.6-6.2$ & 6.2 & $5.2-7.1$ & 5.5 & $4.3-6.7$ & 0.517 & 0.594 & 0.679 & 0.510 \\
\hline Margarine, butter spread and fat spread $(<55 \%)$ & 3.9 & $3.2-4.6$ & 3.2 & $2.3-4.1$ & 2.5 & $1.8-3.3$ & 0.044 & 0.128 & 0.463 & 0.490 \\
\hline Oils & 8.3 & $7.8-8.7$ & 8.9 & $8.3-9.4$ & 7.9 & $7.3-8.6$ & 0.067 & 0.077 & 0.124 & 0.186 \\
\hline
\end{tabular}

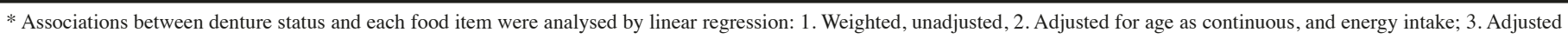

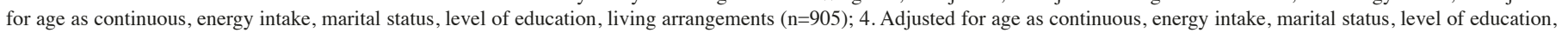
living arrangements, and cognitive function $(n=855)$.

marital status, level of education, living arrangements, and cognitive function (MMSE sum score as continuous). The p-values in tables 2-5 refer to the analysis of variance (or ANOVA) table for the regression analysis (Satterthwaite adjusted F), i.e. p-values show whether the main explanatory variable, e.g. categorised denture status, is statistically significantly associated with the outcome. Examining nonoverlapping confidence intervals for the means, it is possible to see which groups differ statistically significantly from each other. Nutrition intake variables, except E\%, were corrected using the Willett residual method (18). Due to differences in dentition and eating habits between men and women, analyses were made separately for men and women.

\section{Results}

In the study population, the mean number of teeth in dentate participants without removable dentures was about 20 , and in 
Table 3

Weighted means of food consumption ( $\mathrm{g} /$ day) with $95 \%$ confidence intervals $(95 \% \mathrm{CI})$ by denture status in women $(\mathrm{n}=1,215)^{*}$

\begin{tabular}{|c|c|c|c|c|c|c|c|c|c|c|}
\hline \multirow[t]{2}{*}{ FOOD ITEM } & \multicolumn{2}{|c|}{$\begin{array}{l}\text { Dentate, no removable } \\
\text { dentures }(n=455)\end{array}$} & \multicolumn{2}{|c|}{$\begin{array}{l}\text { Dentate with removable } \\
\text { dentures }(n=371)\end{array}$} & \multicolumn{2}{|c|}{$\begin{array}{l}\text { Edentulous with full } \\
\text { dentures }(n=389)\end{array}$} & \multirow[b]{2}{*}{ p-value ${ }^{1}$} & \multirow[b]{2}{*}{ p-value ${ }^{2}$} & \multirow[b]{2}{*}{ p-value ${ }^{3}$} & \multirow[b]{2}{*}{ p-value ${ }^{4}$} \\
\hline & g/day & $95 \% \mathrm{CI}$ & g/day & $95 \% \mathrm{CI}$ & g/day & $95 \% \mathrm{CI}$ & & & & \\
\hline Cereals & 180 & $180-190$ & 190 & $180-200$ & 190 & $180-200$ & 0.101 & 0.249 & 0.293 & 0.180 \\
\hline Rye & 51 & $47-54$ & 54 & $51-57$ & 49 & $45-52$ & 0.041 & 0.039 & 0.042 & 0.022 \\
\hline Wheat & 66 & $62-69$ & 72 & $68-76$ & 75 & $71-80$ & 0.001 & 0.377 & 0.347 & 0.268 \\
\hline Hard bread & 7.8 & $6.6-8.9$ & 7.1 & $6.0-8.3$ & 7.4 & $6.2-8.6$ & 0.721 & 0.600 & 0.899 & 0.595 \\
\hline Vegetables & 330 & $310-350$ & 310 & $290-330$ & 280 & $260-300$ & $<0.001$ & $<0.001$ & $<0.001$ & 0.001 \\
\hline Root vegetables and tubers & 64 & $59-68$ & 67 & $62-72$ & 71 & $64-78$ & 0.167 & 0.999 & 0.954 & 0.835 \\
\hline Potatoes & 180 & $170-190$ & 190 & $180-200$ & 200 & $180-210$ & 0.143 & 0.645 & 0.495 & 0.400 \\
\hline Fruits & 260 & $230-280$ & 200 & $180-200$ & 190 & $160-210$ & $<0.001$ & $<0.001$ & 0.001 & 0.001 \\
\hline Citrus & 69 & $59-80$ & 45 & $36-54$ & 42 & $31-52$ & $<0.001$ & $<0.001$ & 0.006 & 0.005 \\
\hline Apples & 130 & $120-141$ & 99 & $88-110$ & 90 & $76-100$ & $<0.001$ & $<0.001$ & 0.004 & 0.007 \\
\hline Berries & 38 & $35-41$ & 47 & $43-50$ & 48 & $44-52$ & $<0.001$ & 0.011 & 0.004 & 0.004 \\
\hline Juice & 80 & $69-91$ & 70 & $60-80$ & 69 & $59-79$ & 0.209 & 0.082 & 0.446 & 0.630 \\
\hline Fish, fish products, crustaceans and molluses & 52 & $49-55$ & 52 & $47-58$ & 51 & $46-55$ & 0.861 & 0.006 & 0.047 & 0.081 \\
\hline Meat products & 150 & $140-160$ & 150 & $140-160$ & 160 & $150-170$ & 0.180 & 0.747 & 0.783 & 0.555 \\
\hline Red meat & 79 & $74-84$ & 84 & $77-90$ & 91 & $83-99$ & 0.030 & 0.331 & 0.568 & 0.491 \\
\hline Beef & 23 & $21-25$ & 23 & $20-25$ & 25 & $22-28$ & 0.328 & 0.583 & 0.645 & 0.533 \\
\hline Pork & 45 & $42-48$ & 50 & $46-55$ & 55 & $51-60$ & 0.001 & 0.148 & 0.677 & 0.677 \\
\hline Sausages & 28 & $24-31$ & 31 & $26-36$ & 37 & $33-42$ & 0.066 & 0.093 & 0.564 & 0.369 \\
\hline Poultry & 32 & $29-35$ & 28 & $22-33$ & 24 & $19-29$ & 0.011 & 0.042 & 0.101 & 0.183 \\
\hline Eggs & 26 & $24-28$ & 26 & $24-28$ & 29 & $26-32$ & 0.077 & 0.515 & 0.492 & 0.584 \\
\hline Milk & 330 & $300-350$ & 360 & $330-390$ & 440 & $400-470$ & $<0.001$ & 0.003 & 0.017 & 0.056 \\
\hline Soured milk products & 190 & $170-210$ & 210 & $190-230$ & 220 & $200-240$ & 0.138 & 0.624 & 0.525 & 0.824 \\
\hline Cheese & 41 & $38-45$ & 40 & $35-45$ & 32 & $30-35$ & 0.004 & 0.004 & 0.010 & 0.021 \\
\hline Sugary products & 26 & $24-28$ & 29 & $27-31$ & 35 & $32-38$ & $<0.001$ & 0.002 & 0.002 & 0.008 \\
\hline Beverages (-alcohol) & 1600 & $1500-1600$ & 1600 & $1600-1700$ & 1600 & $1600-1700$ & 0.206 & 0.258 & 0.166 & 0.112 \\
\hline \multicolumn{11}{|l|}{ Fat and fat products } \\
\hline Butter and butter spread & 10 & $9.4-11$ & 11 & $10-12$ & 13 & $12-13$ & $<0.001$ & 0.150 & 0.375 & 0.599 \\
\hline \multicolumn{11}{|l|}{ Margarine and fat spread } \\
\hline (FAT) & 4.2 & $3.6-4.8$ & 5.8 & $5.1-6.6$ & 5.4 & $4.6-6.1$ & 0.005 & 0.006 & 0.013 & 0.016 \\
\hline \multicolumn{11}{|l|}{ Margarine, butter spread and } \\
\hline fat spread $(<55 \%)$ & 4.4 & $3.7-5.0$ & 3.9 & $3.1-4.6$ & 3.1 & $2.4-3.7$ & 0.042 & 0.178 & 0.262 & 0.275 \\
\hline Oils & 8.5 & $8.0-8.7$ & 8.4 & $7.9-8.8$ & 8.4 & $7.8-9.0$ & 0.950 & 0.002 & 0.023 & 0.073 \\
\hline
\end{tabular}

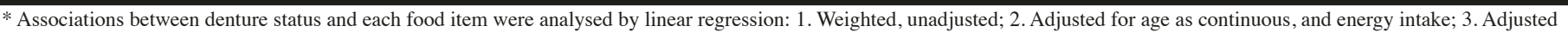

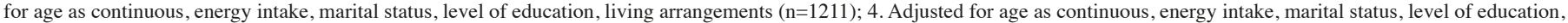
living arrangements, and cognitive function $(n=1120)$.

participants with removable dentures about 10 . There was no essential difference in the number of teeth between genders in the above groups. Furthermore, the proportion of men who had at least 20 teeth was higher than that of women. In all, $30 \%$ of female participants and $22 \%$ of male participants were edentulous full denture wearers.

When viewed socioeconomic background, dentate participants with no removable dentures more often had higher education than dentate participants with removable dentures or full denture wearers. Women who wore full dentures were more often living alone, and men who wore full dentures were in nursing homes or institutions when compared to the dentate group. The dentate participants without removable dentures more often reported their oral health as good, were able to eat dry bread or biscuits without simultaneously drinking water, and they did not have any problems in chewing in comparison to dentate participants with removable dentures who in turn reported better scores in most of the questions related to eating ability than the full denture wearers (Table I). 


\section{JNHA: NUTRITION}

Table 4

Weighted means of nutrient intake (g/day) with $95 \%$ confidence intervals $(95 \% \mathrm{CI})$ by denture status in men $(\mathrm{n}=911)^{*}$

\begin{tabular}{|c|c|c|c|c|c|c|c|c|c|c|}
\hline \multirow[t]{2}{*}{ NUTRITION ITEM } & \multicolumn{2}{|c|}{$\begin{array}{l}\text { Dentate, no removable } \\
\text { dentures }(n=422)\end{array}$} & \multicolumn{2}{|c|}{$\begin{array}{l}\text { Dentate with removable } \\
\text { dentures }(n=286)\end{array}$} & \multicolumn{2}{|c|}{$\begin{array}{l}\text { Edentulous with full } \\
\text { dentures }(n=203)\end{array}$} & \multirow[b]{2}{*}{ p-value ${ }^{1}$} & \multirow[b]{2}{*}{ p-value ${ }^{2}$} & \multirow[b]{2}{*}{ p-value ${ }^{3}$} & \multirow[b]{2}{*}{ p-value ${ }^{4}$} \\
\hline & mean & $95 \% \mathrm{CI}$ & mean & $95 \% \mathrm{CI}$ & mean & $95 \% \mathrm{CI}$ & & & & \\
\hline Energy (Kcal) & 2300 & $2200-2400$ & 2400 & $2300-2500$ & 2300 & $2200-2500$ & 0.222 & 0.316 & 0.281 & 0.150 \\
\hline Fat E\% & 35 & $35-36$ & 36 & $35-36$ & 35 & $34-36$ & 0.137 & 0.214 & 0.152 & 0.144 \\
\hline SFA E $\%^{5}$ & 14 & $14-15$ & 15 & $14-15$ & 15 & $14-14$ & 0.403 & 0.666 & 0.832 & 0.776 \\
\hline MUFA E\% ${ }^{5}$ & 12 & $12-12$ & 12 & $12-12$ & 12 & $11-12$ & 0.023 & 0.093 & 0.092 & 0.108 \\
\hline PUFA E\% ${ }^{5}$ & 5.5 & $5.3-5.6$ & 5.5 & $5.4-5.7$ & 5.1 & $4.9-5.3$ & $<0.001$ & 0.005 & 0.024 & 0.019 \\
\hline Protein E\% & 17 & $17-17$ & 17 & $16-17$ & 16 & $16-16$ & 0.002 & 0.024 & 0.028 & 0.020 \\
\hline Carbohydrates E\% & 45 & $45-46$ & 46 & $45-47$ & 47 & $46-48$ & 0.003 & 0.081 & 0.157 & 0.149 \\
\hline Sucrose $(\mathrm{g})$ & 44 & $42-45$ & 47 & $45-49$ & 49 & $47-52$ & 0.001 & 0.001 & 0.001 & 0.003 \\
\hline Fiber (g) & 25 & $25-26$ & 24 & $24-25$ & 24 & $23-25$ & 0.064 & 0.023 & 0.020 & 0.056 \\
\hline Alcohol E\% & 2.3 & $2.0-2.7$ & 1.8 & $1.4-2.2$ & 1.7 & $1.2-2.3$ & 0.075 & 0.338 & 0.520 & 0.491 \\
\hline Calcium (mg) & 1300 & $1200-1300$ & 1200 & $1200-1300$ & 1200 & $1200-1300$ & 0.213 & 0.072 & 0.031 & 0.022 \\
\hline Carotenes $(\mu \mathrm{g})$ & 10000 & $9400-11000$ & 10000 & $92000-11000$ & 8400 & $7400-9500$ & 0.024 & 0.007 & 0.044 & 0.122 \\
\hline Iron (mg) & 15 & $15-15$ & 15 & $14-15$ & 15 & $14-15$ & 0.408 & 0.443 & 0.558 & 0.592 \\
\hline Potassium (g) & 4.4 & $4.4-4.5$ & 4.3 & $4.3-4.4$ & 4.3 & $4.2-4.4$ & 0.180 & 0.136 & 0.202 & 0.266 \\
\hline Folate $(\mu \mathrm{g})$ & 320 & $320-330$ & 310 & $300-320$ & 310 & $300-320$ & 0.037 & 0.023 & 0.039 & 0.093 \\
\hline Riboflavin (mg) & 2.3 & $2.2-2.3$ & 2.3 & $2.2-2.3$ & 2.3 & $2.2-2.4$ & 0.419 & 0.245 & 0.148 & 0.193 \\
\hline Thiamine (mg) & 1.7 & $1.6-1.7$ & 1.6 & $1.6-1.7$ & 1.6 & $1.6-1.7$ & 0.068 & 0.029 & 0.042 & 0.115 \\
\hline Vitamin A, RE $(\mu \mathrm{g})$ & 1600 & $1500-1700$ & 1600 & $1500-1700$ & 1600 & $1500-1800$ & 0.814 & 0.798 & 0.861 & 0.941 \\
\hline Vitamin B12 $(\mu \mathrm{g})$ & 9.4 & $9.1-9.7$ & 9.6 & $9.1-10$ & 9.7 & $9.1-10$ & 0.569 & 0.657 & 0.714 & 0.753 \\
\hline Vitamin C (mg) & 120 & $110-120$ & 110 & $110-120$ & 100 & $90-110$ & $<0.001$ & $<0.001$ & 0.017 & 0.071 \\
\hline Vitamin $\mathrm{D}(\mu \mathrm{g})$ & 7.0 & $6.7-7.4$ & 7.7 & $7.2-8.1$ & 7.1 & $6.5-7.6$ & 0.071 & 0.057 & 0.068 & 0.034 \\
\hline Vitamin E, TE (mg) & 12 & $12-13$ & 12 & $12-13$ & 11 & $11-12$ & $<0.001$ & $<0.001$ & 0.005 & 0.005 \\
\hline $\mathrm{NaCl}$ (g/day) & 11 & $11-11$ & 11 & $11-11$ & 11 & $10-11$ & 0.945 & 0.934 & 0.821 & 0.832 \\
\hline
\end{tabular}

\section{Food consumption}

In men, food consumption differed between the denture status groups after adjustments for age, total energy intake, marital status, level of education, living arrangements and cognitive function for vegetables $(\mathrm{p}=0.013)$, apples $(\mathrm{p}=$ $0.008)$, fish and fish products $(\mathrm{p}=0.004)$, cheese $(\mathrm{p}=0.003)$ and sugary products $(\mathrm{p}=0.012)$. The differences were most evident between dentate men without removable dentures and edentate men with full dentures. However, the use of fish made an exception, as dentate men used less fish than dentate men with removable dentures (Table II).

In women, food consumption differed between the denture status groups after adjustments for age, total energy intake, marital status, level of education, living arrangements and cognitive function for rye $(p=0.022)$, vegetables $(p=0.001)$, fruits $(\mathrm{p}=0.001)$ (citrus $(\mathrm{p}=0.005)$, apples $(\mathrm{p}=0.007)$ and berries $(\mathrm{p}=0.004))$, cheese $(\mathrm{p}=0.021)$, sugary products $(\mathrm{p}$ $=0.008)$ and margarine and fat spreads $(p=0.016)$. (Table III). Differences between dentate women without removable dentures and edentate women with full dentures were not as categorical as in men, although the use of vegetables, cheese and sugary products differed between these groups. Dentate women without removable dentures used more fruits (citruses and apples), but less berries than dentate women with removable dentures and edentate women with full dentures. Dentate women with removable dentures used more rye and margarine and fat spreads than the other two groups. (Table III).

\section{Nutrient intake}

Intake of polyunsaturated fatty acids $(\mathrm{E} \%)(\mathrm{p}=0.019)$, protein $(\mathrm{E} \%)(\mathrm{p}=0.020)$, sucrose $(\mathrm{g} / \mathrm{day})(\mathrm{p}=0.003)$, calcium $(\mathrm{mg} /$ day $)(\mathrm{p}=0.022)$ and vitamins $\mathrm{D}(\mathrm{p}=0.034)$ and $\mathrm{E}(\mathrm{p}=$ 0.005 ) differed in men between denture status groups after adjustments for age, total energy intake, marital status, level of education, living arrangements and cognitive function. (Table IV). The differences were most evident between dentate men without removable dentures and edentate men with full dentures. 
Table 5

Weighted means of nutrient intake ( $\mathrm{g} /$ day) with $95 \%$ confidence intervals $(95 \% \mathrm{CI})$ by denture status in women $(\mathrm{n}=1,215)^{*}$

\begin{tabular}{|c|c|c|c|c|c|c|c|c|c|c|}
\hline \multirow[t]{2}{*}{ NUTRITION ITEM } & \multicolumn{2}{|c|}{$\begin{array}{l}\text { Dentate, no removable } \\
\text { dentures }(n=422)\end{array}$} & \multicolumn{2}{|c|}{$\begin{array}{l}\text { Dentate with removable } \\
\text { dentures }(n=286)\end{array}$} & \multicolumn{2}{|c|}{$\begin{array}{l}\text { Edentulous with full den- } \\
\text { tures }(n=203)\end{array}$} & \multirow[b]{2}{*}{ p-value ${ }^{1}$} & \multirow[b]{2}{*}{ p-value ${ }^{2}$} & \multirow[b]{2}{*}{ p-value ${ }^{3}$} & \multirow[b]{2}{*}{ p-value ${ }^{4}$} \\
\hline & mean & $95 \% \mathrm{CI}$ & mean & $95 \% \mathrm{CI}$ & mean & $95 \% \mathrm{CI}$ & & & & \\
\hline Energy (Kcal) & 2100 & $2000-2200$ & 2200 & $2100-2300$ & 2300 & $2200-2400$ & 0.001 & 0.027 & 0.019 & 0.052 \\
\hline Fat E\% & 35 & $35-36$ & 35 & $34-35$ & 35 & $35-36$ & 0.604 & 0.669 & 0.597 & 0.622 \\
\hline SFA E $\%^{5}$ & 14 & $14-14$ & 14 & $14-15$ & 15 & $14-15$ & 0.002 & 0.111 & 0.287 & 0.171 \\
\hline MUFA E\% ${ }^{5}$ & 12 & $12-12$ & 12 & $12-12$ & 12 & $12-12$ & 0.548 & 0.580 & 0.445 & 0.641 \\
\hline PUFA E\% ${ }^{5}$ & 5.8 & $5.7-6.0$ & 5.6 & $5.5-5.7$ & 5.4 & $5.3-5.5$ & $<0.001$ & 0.001 & 0.022 & 0.036 \\
\hline Protein E\% & 18 & $17-18$ & 17 & $17-18$ & 17 & $17-17$ & 0.002 & 0.074 & 0.083 & 0.105 \\
\hline Carbohydrates E\% & 46 & $46-47$ & 46 & $47-48$ & 47 & $47-48$ & 0.015 & 0.045 & 0.054 & 0.075 \\
\hline Sucrose (g) & 44 & $43-46$ & 48 & $46-50$ & 50 & $48-52$ & $<0.001$ & $<0.001$ & $<0.001$ & $<0.001$ \\
\hline Fiber (g) & 29 & $28-29$ & 28 & $27-29$ & 26 & $26-27$ & $<0.001$ & $<0.001$ & 0.001 & $<0.001$ \\
\hline Alcohol E\% & 0.97 & $0.83-1.1$ & 0.45 & $0.37-0.53$ & 0.37 & $0.29-0.45$ & $<0.001$ & $<0.001$ & $<0.001$ & $<0.001$ \\
\hline Calcium (mg) & 1400 & $1300-1400$ & 1400 & $1300-1400$ & 1400 & $1300-1400$ & 0.782 & 0.788 & 0.805 & 0.447 \\
\hline Carotens $(\mu \mathrm{g})$ & 12000 & $11000-13000$ & 12000 & $11000-12000$ & 11000 & $10000-12000$ & 0.100 & 0.001 & 0.001 & $<0.001$ \\
\hline Iron (mg) & 15 & $15-16$ & 15 & $15-16$ & 15 & $14-15$ & $<0.001$ & 0.025 & 0.130 & 0.127 \\
\hline Potassium (g) & 4.9 & $4.8-5.0$ & 4.7 & $4.7-4.8$ & 4.6 & $4.5-4.7$ & $<0.001$ & $<0.001$ & 0.006 & 0.046 \\
\hline Folate $(\mu \mathrm{g})$ & 360 & $350-370$ & 350 & $340-360$ & 330 & $320-340$ & $<0.001$ & $<0.001$ & 0.001 & 0.001 \\
\hline Riboflavin (mg) & 2.5 & $2.4-2.5$ & 2.5 & $2.4-2.5$ & 2.5 & $2.4-2.5$ & 0.935 & 0.962 & 0.925 & 0.683 \\
\hline Thiamine (mg) & 1.8 & $1.7-1.8$ & 1.7 & $1.7-1.7$ & 1.7 & $1.6-1.7$ & 0.014 & 0.047 & 0.084 & 0.112 \\
\hline Vitamin A, RE $(\mu \mathrm{g})$ & 1900 & $1800-2000$ & 1900 & $1800-2000$ & 1800 & $1700-1900$ & 0.456 & 0.493 & 0.517 & 0.453 \\
\hline Vitamin B12 $(\mu \mathrm{g})$ & 9.9 & $9.6-10$ & 9.9 & $9.4-10$ & 9.7 & $9.3-10$ & 0.656 & 0.298 & 0.408 & 0.438 \\
\hline Vitamin C (mg) & 170 & $160-180$ & 150 & $140-150$ & 140 & $130-140$ & $<0.001$ & $<0.001$ & $<0.001$ & $<0.001$ \\
\hline Vitamin $\mathrm{D}(\mu \mathrm{g})$ & 7.3 & $7.2-7.8$ & 7.5 & $7.1-7.9$ & 7.0 & $6.6-7.4$ & 0.093 & 0.003 & 0.017 & 0.016 \\
\hline Vitamin E, TE (mg) & 14 & $13-14$ & 13 & $13-14$ & 12 & $12-13$ & $<0.001$ & $<0.001$ & 0.001 & $<0.001$ \\
\hline $\mathrm{NaCl}$ (g/day) & 11 & $11-11$ & 11 & $11-11$ & 11 & $11-11$ & 0.092 & 0.082 & 0.071 & 0.199 \\
\hline
\end{tabular}

In women the intake of polyunsaturated fatty acids $(\mathrm{E} \%)$ $(\mathrm{p}=0.036)$, sucrose $(\mathrm{g} /$ day $)(\mathrm{p}=<0.001)$, fibre $(\mathrm{g} / \mathrm{day})(\mathrm{p}$ $=<0.001)$, alcohol $(\mathrm{E} \%)(\mathrm{p}=<0.001)$, potassium $(\mathrm{g} / \mathrm{day})(\mathrm{p}$ $=0.046)$, folate (ug/day) $(\mathrm{p}=0.001)$, and vitamins $\mathrm{C}(\mathrm{p}=$ $<0.001)), \mathrm{D}(\mathrm{p}=0.016)$ and $\mathrm{E}(\mathrm{p}=<0.001)$ differed between denture status groups after adjustments for age, total energy intake, marital status, level of education, living arrangements and cognitive function (Table V). The differences were mainly observed between dentate women without removable dentures and edentate women with full dentures. However, dentate women without removable dentures showed a higher intake of alcohol and vitamin $\mathrm{C}$ than dentate women with removable dentures or edentate women with full dentures (Table V).

\section{Discussion}

One of the most important findings of this study was that the use of full dentures appears to be associated with food selection. Both men and women who wore full dentures or removable dentures consumed less fruits and vegetables than dentate participants without any removable dentures. It was found that edentulous full denture wearers had an unhealthier diet, used more sugary products and used fats less favourably than dentate subjects with no removable dentures. This result has not been reported earlier. In the dentate individuals, differences between the two groups (own dentition with removable dentures, own dentition with no removable dentures) were fairly small. Statistically significant differences between these two groups were mostly observed in women.

Only a few population studies have focused on whether denture status is related to food consumption including nutrient intake $(1,2,3,4)$. When comparing the results of this study to the results of other population studies, it can be seen that the findings are in line with reports from the UK and USA $(1,3$, 4). In relation to nutrient intake, the results of this study are in agreement with a study conducted in an elderly UK population; poorer intake of vitamins, such as $\mathrm{C}$ and $\mathrm{E}$ and less dietary fibre when full denture wearers were compared with their dentate 


\section{JNHA: NUTRITION}

counterparts (4). Lower consumption of vegetables and the lower intake of fibres among edentulous persons in comparison to individuals with their own natural teeth have also been reported in the USA (NHANES) (1).

The mechanisms underlying differences in nutrient intake and food consumption between full denture wearers, dentate subjects with removable dentures and dentate individuals with no removable dentures remain unresolved. However, it has been reported previously that denture wearers have an impaired chewing ability and they swallow less fragmented food boli than people with their own natural teeth (19). Based on this and the findings of the present study, it could be speculated that denture wearers find eating problematic and therefore choose foods that are easier to chew but which at the same time are unhealthier. In addition to food selection, it is possible that there are also age related differences in the absorption of nutrients, for instance as described by Remond (20).

One strength of this study is that the results are based on a nationally representative sample with a high response rate also in the older age groups. This means that the results can be generalised to the total Finnish population and also to other populations, naturally, most easily to those of Caucasian origin and populations with a similar level of oral health and similar kind of dental health system. Another strength is that the results are based on a large set of food items and nutrients, which provides a better picture of the entire diet. The food frequency questionnaire, which is based on selfadministered questionnaires, has been shown to be a valid method for assessing habitual/long-term food consumption (14). In any assessment that evaluates the healthiness of the diet, it is important to adjust nutrient intake to the total energy intake (21). Total energy intake was taken into account in this study by using the Willett residual method (18). An obvious weakness is that it does not allow us to make any causal interpretations on the effects of denture status on food consumption or nutrient intake; this is due to the descriptive nature of the study.

Although full denture wearers had the highest intake of carbohydrates, all three denture status groups were within the Nordic Council's nutrient recommendations for carbohydrates (45-60 E\%) (22). There was a difference in fibre intake between men and women, i.e. all male denture status groups were below the recommended level whereas females consumed enough fibre irrespective of their denture status. The results suggest that in order to achieve the level that has been recommended for fibre intake (25-35 g/day), it would be necessary to increase the amount of vegetables and carbohydrate foods rich in fibre by limiting the amount of sugars and fat in the diet (22). This change in diet would not only have a beneficial effect on oral health but would also reduce problems related to obesity.

The quality of the fat that is consumed is important for cardiovascular health. In this study population, there was a significant difference between the denture status groups in their intakes of polyunsaturated fatty acids. Among participants who wore full dentures, the intake of polyunsaturated fatty acids as a percentage of the total energy intake barely reached the recommended lowest level (5-10 E\%) (22). The intake of saturated fatty acids was slightly, although not statistically significantly, higher among edentulous full denture wearers and dentate participants who used removable dentures in comparison to dentate participants with no removable dentures. From the cardiovascular point of view, it is important to note that the overall intake of saturated fatty acids among all participants was higher than the recommended level (10 E\%), even though total fat intake in the study population remained within the recommended level at the population level (25-40 E\%) (22).

This study showed that the intake of nearly all vitamins was lower among edentate full denture wearers than among dentate individuals with or without removable dentures. It should be noted that in all denture status groups, the intake of all vitamins was above the recommended level except for vitamin $\mathrm{D}$, where intake was clearly below the recommended level (10 ug/day) among all participants, both dentate and edentate (22). Fortification of dairy products with vitamin $\mathrm{D}$ is conducted in many countries because the vitamin D content is inherently rather low in most foods.

Masticatory function appears to be important for older people, who are more likely to suffer from health consequences related to dietary restrictions (23). Dietary changes following tooth loss and poor dental status have been suggested to be associated with an increased risk of developing chronic diseases as well as with mortality $(7,24)$.

Based on the results, we suggest that edentulism in an old person is one factor among others that may lead to malnutrition, which according to WHO (World Health Organisation) is defined as a cellular imbalance between supply of nutrients and energy and the body's demand for them to ensure growth, maintenance and specific functions (25). In addition to edentulism, it is worth remembering that there are several physiological changes in an aging person's body that affect eating habits and food choices. These include, for example, that older people feel less hungry than their younger counterparts due to hormonal changes (26) and they may also suffer from "dry mouth syndrome", which alters the perception of taste and smell $(27,29)$.

It has previously been reported that the oral health-related quality of life is related to the risk of developing malnutrition (29) and that a sufficient dentition comprises 20 teeth with 9-10 occlusal units (30). In their clinical practice, dentists should especially ask older people with insufficient dentition and denture wearers who report that their denture fit is problematic, about their ability to eat hard or chewy foods (31) and give advice on how to improve their oral health and, if necessary, refer patients to nutritional counselling. In addition, replacement of extracted teeth should be planned to avoid deterioration of masticatory ability. 


\section{NUTRITION AND DENTURE USE}

\section{Conclusions}

Wearing full dentures appears to be associated with unhealthier food choices, lower consumption of some foodstuffs and lower intake of certain nutrients when compared to the food choices of dentate persons. More studies will be required to elucidate the role of oral health on nutrition, especially other clinical oral health outcomes with longitudinal study designs.

Acknowledgements: The present study is part of the Health 2000 Survey, organized by the National Institute for Health and Welfare (THL), the former Public Health Institute (KTL) of Finland (http://www.terveys2000.fi/indexe.html), and partly supported by the Finnish Dental Society Apollonia and the Finnish Dental Association. The personal grants to Leena Jauhiainen from the Finnish Dental Association and the Dental Society of Northern Savo are acknowledged.

Conflicts of interest: None to declare.

Ethical Standards: Each participant gave their written, informed consent. Approval of the Ethics Committee of Epidemiology and Public Health in the Hospital District of Helsinki and Uusimaa was also obtained.

\section{References}

1. Nowjack-Raymer RE, Sheiham A. Association of edentulism and diet and nutrition in US adults. J Dent Res 2003;82:123-126

2. Ranta K, Tuominen R, Paunio I, Seppänen R. Dental status and intake of food items among an adult Finnish population. Gerodontics 1988;4:32-35

3. Sheiham A, Steele JG, Marcenes W, Finch S, Walls AW. The impact of oral health on stated ability to eat certain foods; findings from the National Diet and Nutrition Survey of Older People in Great Britain. Gerodontology 1999;16:11-20

4. Sheiham A, Steele JG, Marcenes W, Lowe C, Finch S, Bates CJ et al. The relationship among dental status, nutrient intake, and nutritional status in older people. J Dent Res 2001;80:408-413.

5. Sahyoun NR, Krall E. Low dietary quality among older adults with self-perceived illfitting dentures. J Am Diet Assoc 2003;103:1494-1499

6. Greksa LP, Parraga IM, Clark CA. The dietary adequacy of edentulous older adults. J Prosthet Dent 1995;73:142-145

7. Appollonio I, Carabellese C, Frattola A, Trabucchi M. Influence of dental status on dietary intake and survival in community-dwelling elderly subjects. Age Ageing 1997;26:445-456

8. Papas AS, Palmer CA, Rounds MC, Herman J, McGandy RB, Hartz SC et al. Longitudinal relationships between nutrition and oral health. Ann N Y Acad Sci 1989;561:124-142

9. Joshipura KJ, Willett WC, Douglass CW. The impact of edentulousness on food and nutrient intake. J Am Dent Assoc 1996;127:459-467

10. Shimazaki Y, Soh I, Saito T, Yamashita Y, Koga T, Miyazaki H, Takehara T. Influence of dentition status on physical disability, mental impairment, and mortality in institutionalized elderly people. J Dent Res. 2001;80: 340-345
11. Saarela RK, Soini H, Hiltunen K, Muurinen S, Suominen M, Pitkälä K. Dentition status, malnutrition and mortality among older service housing residents. J Nutr Health Aging. 2014;8: 34-38

12. Preshaw PM, Walls AW, Jakubovics NS, Moynihan PJ, Jepson NJ, Loewy Z. Association of removable partial denture use with oral and systemic health. J Dent 2011;39:711-719

13. Aromaa A, Koskinen S, eds. Health and functional capacity in Finland, Baseline Results of the Health 2000 Health Examination Survey. Publications of the National Public Health Institute B12/2004. Hakapaino Oy, Helsinki 2004.

14. Suominen-Taipale L, Nordblad A, Vehkalahti M, Aromaa A, eds. Oral Health in the Finnish Adult Population, Health 2000 Survey. Publications of the National Public Health Institute B25/2008. Hakapaino Oy, Helsinki, 2008

15. Männistö S, Virtanen M, Mikkonen T, Pietinen P. Reproducibility and validity of a food frequency questionnaire in a case-control study on breast cancer. J Clin Epidemiol 1996;49:401-409

16. Männistö S, Montonen J, Pietinen P, Knekt P. Dietary questionnaire. In: Heistaro S, eds. Methodology Report, Health 2000 Survey, pp. 121-123. Publications of the National Public Health Institute B26/2008. Hakapaino Oy, Helsinki, 2008.

17. Folstein MF, Folstein SE, McHugh PR. "'Mini-mental state'. A practical method for grading the cognitive state of patients for the clinician. J Psychiatr Res 1975;12:189_ 198

18. Willett WC, Howe GR, Kushi LH. Adjustment for total energy intake in epidemiologic studies. Am J Clin Nutr 1997;65:1220S-1228S

19. Yven C, Bonnet L, Cormier D, Monier S, Mioche L. Impaired mastication modifies the dynamics of bolus formation. Eur J Oral Sci 2006;114:184-190

20. Rémond D, Machebeuf M, Yven C, Buffière C, Mioche L, Mosoni L et al. Postprandial whole-body protein metabolism after a meat meal is influenced by chewing efficiency in elderly subjects. Am J Clin Nutr 2007;85:1286-1292

21. Willett W. Implications of Total Energy Intake for Epidemiologic Analyses. In: Nutritional Epidemiology, 3rd Ed. Oxford University Press, New York, 2013

22. Nordic Council of Ministers 2013. Nordic Nutrition Recommendations 2012, Part 1. Summary, principles and use. 5th edition, 2012

23. Rémond D, Shahar DR, Gille D, Pinto P, Kachal J, Peyron MA, Dos Santos CN Walther B, Bordoni A, Dupont D, Tomás-Cobos L, Vergères G. Understanding the gastrointestinal tract of the elderly to develop dietary solutions that prevent malnutrition. Oncotarget. 2015;6:13858-13898.

24. Hung HC, Willett W, Ascherio A, Rosner BA, Rimm E, Joshipura KJ. Tooth loss and dietary intake. J Am Dent Assoc 2003;134:1185-1192

25. de Onís M, Monteiro C, Akré J, Glugston G. The worldwide magnitude of proteinenergy malnutrition: an overview from the WHO Global Database on Child Growth. Bull World Health Organ 1993;71:703-712

26. Susuki K, Simpson KA, Minnion JS, Shillito JC, Bloom SR (2010). The role of gut hormones and the hypothalamus in appetite regulation. Endocr J 2010;57:359-372

27. Schiffman SS. Taste and smell losses in normal aging and disease. JAMA 1997;278:1357-1362

28. Närhi TO, Meurman JH, Ainamo A. Xerostomia and hyposalivation: causes, consequences and treatment in the elderly. Drugs Aging 1999;5:103-116.

29. Gil-Montoya JA, Subirá C, Ramón JM, González-Moles MA. Oral health-related quality of life and nutritional status. J Public Health Dent 2008;68:88-93

30. Gotfredsen K, Walls AW. What dentition assures oral function? Clin Oral Implants Res 2007; 18:34-45

31. Iwasaki M, Taylor GW, Manz MC, Yoshihara A, Sato M, Muramatsu K et al. Oral health status: relationship to nutrient and food intake among 80-year-old Japanese adults. Community Dent Oral Epidemiol 2014;42:441-450 